\title{
Gaussian Wave-functional Approach in Thermofield Dynamics
}

\author{
Wen-Fa Lu \\ CCAST(World Laboratory) P.O. Box 8730, Beijing, 100080, \\ and \\ Physics of Department, Shanghai Jiao Tong University, Shanghai 200030, China
}

(August 20, 2018)

\begin{abstract}
The Gaussian wavefunctional approach is developed in thermofield dynamics. We manufacture thermal vacuum wavefunctional, its creation as well as annihilation operators, and accordingly thermo-particle excited states. For a $(D+1)$-dimensional scalar field system with an arbitrary potential whose Fourier representation exists in a sense of tempered distributions, we calculate the finite temperature Gaussian effective potential (FTGEP), one- and two-thermo-particle-state energies. The zero-temperature limit of each of them is just the corresponding result in quantum field theory, and the FTGEP can lead to the same one of each of some concrete models as calculated by the imaginary time Green function.
\end{abstract}

The Gaussian wave-functional approach (GWFA ) [1] has become an important non-perturbative tool of quantum field theory and condensed matter physics [2, 3] since Stevenson's advocation in 1985 [4]. It is simple, feasible, effective, and can reveal useful non-perturbative information (at least qualitatively). A result from the GWFA can give a helpful guidance or be taken as a good start for a further investigation [5]. Moreover, this approach is often used for some novel investigations or new ideas [6]. So the GWFA was inevitably generalized to finite temperature field theory, by a direct calculation of partition function from the first principle or by calculating the real-time or imaginary-time Green functions [7]. However, thermofield dynamics [8], the third formalism of finite temperature field theory, has its own advantages over the other two formalisms : the closed time path and the imaginary time formalisms [9]. It can reply to some questions which are not possible within the framework of the closed time path formalism, such as the structure of the thermal vacuum, the nature of the Goldstone states, and so on [9]. Also, Thermofield dynamics can describe very naturally the time development for quantities near equilibrium [9]. Hence it is worth while generalizing the GWFA to thermofield dynamics. In 1986, through considering the $\lambda \phi_{(0+1)}^{4}$ theory, I. Roditi gave the quantummechanical finite temperature Gaussian effective potential ( FTGEP ) within the framework of thermofield dynamics [10]. Recently, A. Mishra and H. Mishra calculated the FTGEP of the $\lambda \phi_{(3+1)}^{4}$ theory by the combination of the zero-temperature Bogoliubov and the thermal Bogoliubov transformation techniques ( TDBTT ) [11. In this letter, we intend to develop Roditi's pioneering work and establish the GWFA of thermofield dynamics ( TDGWFA ) in $(D+1)$ dimensions.

We consider a relatively general model with the Lagrangian density

$$
\mathcal{L}=\frac{1}{2} \partial_{\mu} \phi_{x} \partial^{\mu} \phi_{x}-V\left(\phi_{x}\right)
$$

where $\phi_{x} \equiv \phi(\vec{x})$, and the potential $V\left(\phi_{x}\right)$ has a Fourier reprensentation in a sense of tempered distributions [12]. Potentials of many models, such as various polynomial models, sine-Gordon and sinh-Gordon models, have this property. We suppose that the field system Eq.(1) is immerged into a thermal reservoir with a fixed temperature $T=\frac{1}{k_{b} \beta}$ ( $k_{b}$ represents the Boltzmann constant), i.e., we shall consider canonical ensemble. (As for the Grand canonical ensemble, the generalization is straight foreward.) After manufacturing the thermal vacuum wavefunctional and the thermal particle creation as well as annihilation operators, we shall calculate the FTGEP, one- and twothermo-particle energies, and then conclude this letter by a brief discussion.

By the way, thermofield dynamics and the GWFA in quantum field theory will be not introduced in this letter, and good exposition of them can be found in Refs. [8,9] and refs. [1] 3], respectively.

Let us begin with quantum field theory. In the fixed-time functional Schrödinger picture, the canonical conjugate operater to $\phi_{x}$ is $\Pi_{x} \equiv-i \frac{\delta}{\delta \phi_{x}}$ with the commutator $\left[\phi_{x}, \Pi_{y}\right]=i \delta(x-y)$. For the convenience of renormalizing the theory of the system Eq.(1) later, we shall use the following normal-ordered Hamiltonian density with respect to any normal-ordering mass $Q[13]$

$$
\mathcal{N}_{Q}\left[\mathcal{H}_{x}\right]=\frac{1}{2} \Pi_{x}^{2}+\frac{1}{2}\left(\nabla \phi_{x}\right)^{2}-\frac{1}{2} I_{0}\left[Q^{2}\right]+\frac{1}{4} Q^{2} I_{1}\left[Q^{2}\right]+\mathcal{N}_{Q}\left[V\left(\phi_{x}\right)\right]
$$

where $\nabla$ represents the gradient operator in $D$-dimensional $\vec{x}$-space, $p=|\vec{p}|$, and $I_{n}\left[Q^{2}\right]=\int \frac{d^{D} \vec{p}}{(2 \pi)^{D}} \frac{\sqrt{p^{2}+Q^{2}}}{\left(p^{2}+Q^{2}\right)^{n}}$. As for $\mathcal{N}_{Q}\left[V\left(\phi_{x}\right)\right]$, making the Fourier transformation ( at least in a sense of tempered distributions [12] ) and using the Baker-Hausdorff formula, one can have 


$$
\mathcal{N}_{Q}\left[V\left(\phi_{x}\right)\right]=\int_{-\infty}^{\infty} \frac{d \Omega}{\sqrt{2 \pi}} \tilde{V}(\Omega) e^{i \Omega \phi_{x}} e^{\frac{1}{4} \Omega^{2} I_{1}\left[Q^{2}\right]}
$$

Similar to the GWFA in quantum field theory [1, 3], we take following Gaussian wavefunctional as the quantum vacuum ansatz of $T=0$ field system Eq.(1)

$$
\mid \varphi>=N_{f} \exp \left\{-\frac{1}{2} \int_{x, y}\left(\phi_{x}-\varphi_{x}\right) f_{x y}\left(\phi_{y}-\varphi_{y}\right)\right\},
$$

where $\varphi_{x}$ and $f_{x y}$ are variational parameter functions. $N_{f}$ is some normalization constant, and depends upon $f_{x y}$. Due to the translational invariance of vacuum, it is required that $f_{x y}=f(x-y)$. Also, in view of the symmetric form of the vacuum, we assume that $f_{x y}=f_{y x}$. Besides, the inverse $f_{x y}^{-1}$ of $f_{x y}$ has to exist. It is easy to show that $\varphi_{x}$ is the expectation value of the field operator with respect to the above trial vacuum $|\varphi\rangle$. The creation and the annihilation operators to the vacuum Eq.(4) can be constructed as [1,3]

$$
A_{f}(\vec{p})=\left(\frac{1}{2(2 \pi)^{D} f(\vec{p})}\right)^{1 / 2} \int_{x} e^{-i \vec{p} \cdot \vec{x}}\left[f(\vec{p})\left(\phi_{x}-\varphi_{x}\right)+i \Pi_{x}\right]
$$

and

$$
A_{f}^{\dagger}(\vec{p})=\left(\frac{1}{2(2 \pi)^{D} f(\vec{p})}\right)^{1 / 2} \int_{x} e^{i \vec{p} \cdot \vec{x}}\left[f(\vec{p})\left(\phi_{x}-\varphi_{x}\right)-i \Pi_{x}\right]
$$

respectively, where $f(\vec{p})$ is the Fourier transform of $f_{x y}$. Of course, one can check the relations $\left[A_{f}(\vec{p}), A_{f}^{\dagger}\left(\vec{p}^{\prime}\right)\right]=$ $\delta\left(\vec{p}^{\prime}-\vec{p}\right)$ and $A_{f}(\vec{p}) \mid \varphi>=0$.

In order to calculate average value of any physical quantity, thermofield dynamics needs the tilde system, the copy of the $T=0$ field system. Corresponding to the above, the tilde system has the normal-ordered Hamiltonian density

$$
\mathcal{N}_{Q}\left[\tilde{\mathcal{H}}_{x}\right]=\frac{1}{2} \tilde{\Pi}_{x}^{2}+\frac{1}{2}\left(\nabla \tilde{\phi}_{x}\right)^{2}-\frac{1}{2} I_{0}\left[Q^{2}\right]+\frac{1}{4} Q^{2} I_{1}\left[Q^{2}\right]+\mathcal{N}_{Q}\left[V\left(\tilde{\phi}_{x}\right)\right]
$$

and the quantum vacuum

$$
\mid \tilde{\varphi}>=N_{f} \exp \left\{-\frac{1}{2} \int_{x, y}\left(\tilde{\phi}_{x}-\varphi_{x}\right) f_{x y}\left(\tilde{\phi}_{y}-\varphi_{y}\right)\right\}
$$

where $\tilde{\Pi}_{x} \equiv i \frac{\delta}{\delta \tilde{\phi}_{x}}$ conjugates to the tilde field operator $\tilde{\phi}_{x}$ with the commutator $\left[\tilde{\phi}_{x}, \tilde{\Pi}_{y}\right]=-i \delta(x-y)$. The appearance of the additional minus in the commutation relation is due to the rule of the tilde operation [8.9.9. Note that it is not necessary to distinguish the ordinary real function and average values of the $T=0$ system with those of its tilde system. From the rule of the tilde operation, the annihilation and the creation operators to the tilde vacuum Eq.(8) read

$$
\tilde{A}_{f}(\vec{p})=\left(\frac{1}{2(2 \pi)^{D} f(\vec{p})}\right)^{1 / 2} \int_{x} e^{i \vec{p} \cdot \vec{x}}\left[f(\vec{p})\left(\tilde{\phi}_{x}-\varphi_{x}\right)-i \tilde{\Pi}_{x}\right]
$$

and

$$
\tilde{A}_{f}^{\dagger}(\vec{p})=\left(\frac{1}{2(2 \pi)^{D} f(\vec{p})}\right)^{1 / 2} \int_{x} e^{-i \vec{p} \cdot \vec{x}}\left[f(\vec{p})\left(\tilde{\phi}_{x}-\varphi_{x}\right)+i \tilde{\Pi}_{x}\right] .
$$

Of course, the relations $\left[\tilde{A}_{f}(\vec{p}), \tilde{A}_{f}^{\dagger}\left(\vec{p}^{\prime}\right)\right]=\delta\left(\vec{p}^{\prime}-\vec{p}\right)$ and $\tilde{A}_{f}(\vec{p}) \mid \tilde{\varphi}>=0$ hold. Additionally, each operator of the tilde system commutes with that of the original system.

Basing on the $T=0$ field system and its tilde partner, now we take the following wavefunctional as the thermal vacuum ansatz of the $T \neq 0$ field system

$$
\left|\varphi, \beta>=\exp \left\{i \int_{x, y}\left[\left(\phi_{x}-\varphi_{x}\right) g_{x y}(\beta) \tilde{\Pi}_{y}-\Pi_{x} g_{x y}(\beta)\left(\tilde{\phi}_{y}-\varphi_{y}\right)\right]\right\}\right| \varphi \tilde{\varphi}>,
$$

where $\mid \varphi \tilde{\varphi}>$ represents the direct product of $\mid \varphi>$ and $\mid \tilde{\varphi}>$, i.e., 


$$
\mid \varphi \tilde{\varphi}>=N_{f}^{2} \exp \left\{-\frac{1}{2} \int_{x, y}\left[\left(\phi_{x}-\varphi_{y}\right) f_{x y}\left(\phi_{y}-\varphi_{y}\right)+\left(\tilde{\phi}_{x}-\varphi_{x}\right) f_{x y}\left(\tilde{\phi}_{y}-\varphi_{y}\right)\right]\right\} .
$$

Analogous with $f_{x y}, g_{x y}(\beta)=g_{y x}(\beta)=g(x-y, \beta)$, and it is also a temperature-dependent variational parameter function to be determined. When it acts on the thermal vacuum wavefunctional Eq.(11), any operator of the original system works only for quantities of the original system itself. So does that of the tilde system. According to Ref. [14], one can have ( here the Baker-Hausdorff formula is invalid )

$$
\begin{aligned}
& \exp \left\{i \int_{x, y}\left[\left(\phi_{x}-\varphi_{x}\right) g_{x y}(\beta) \tilde{\Pi}_{y}-\Pi_{x} g_{x y}(\beta)\left(\tilde{\phi}_{y}-\varphi_{y}\right)\right]\right\} \\
= & \exp \left\{-\int_{x p y}^{\prime} \operatorname{th}(g(\vec{p}, \beta)) \Pi_{x}\left(\tilde{\phi}_{y}-\varphi_{y}\right)\right\} \\
& \cdot \exp \left\{\int_{x p y}^{\prime} \ln (\operatorname{ch}(g(\vec{p}, \beta)))\left[\left(\phi_{x}-\varphi_{x}\right) \Pi_{y}+\left(\tilde{\phi}_{y}-\varphi_{x}\right) \tilde{\Pi}_{x}\right]\right\} \\
& \cdot \exp \left\{\int_{x p y}^{\prime} \operatorname{th}(g(\vec{p}, \beta))\left(\phi_{x}-\varphi_{x}\right) \tilde{\Pi}_{y}\right\},
\end{aligned}
$$

where $\int_{x p y}^{\prime} \equiv i \int_{x, y} \int \frac{d^{D} \vec{p} e^{i \vec{p} \cdot(\vec{x}-\vec{y})}}{(2 \pi)^{D}}$, and $g(p, \beta)$ is the Fourier transform of the function $g_{x y}(\beta)$. Hence one can write Eq.(11) as

$$
\begin{aligned}
\mid \varphi, \beta>= & N_{f}^{2} \exp \left\{-\frac{i}{2} \int_{x p y}^{\prime}\left[\left(\phi_{x} \operatorname{ch}(g(\vec{p}, \beta))-\tilde{\phi}_{x} \operatorname{sh}(g(\vec{p}, \beta))-\varphi_{x}(1-\operatorname{sh}(g(\vec{p}, \beta)))\right)\right.\right. \\
& \cdot f(\vec{p})\left(\phi_{y} \operatorname{ch}(g(\vec{p}, \beta))-\tilde{\phi}_{y} \operatorname{sh}(g(\vec{p}, \beta))-\varphi_{y}(1-\operatorname{sh}(g(\vec{p}, \beta)))\right) \\
& +\left(\tilde{\phi}_{x} \operatorname{ch}(g(\vec{p}, \beta))-\phi_{x} \operatorname{sh}(g(\vec{p}, \beta))-\varphi_{x}(1-\operatorname{th}(g(\vec{p}, \beta))+\operatorname{sh}(g(\vec{p}, \beta)) \operatorname{th}(g(\vec{p}, \beta)))\right) \\
& \left.\left.\cdot f(\vec{p})\left(\tilde{\phi}_{y} \operatorname{ch}(g(\vec{p}, \beta))-\phi_{y} \operatorname{sh}(g(\vec{p}, \beta))-\varphi_{y}(1-\operatorname{th}(g(\vec{p}, \beta))+\operatorname{sh}(g(\vec{p}, \beta)) \operatorname{th}(g(\vec{p}, \beta)))\right)\right]\right\} .
\end{aligned}
$$

This wavefunctional is just Gaussian for $\phi$ or for $\tilde{\phi}$.

For the thermal vacuum Eq.(11) or (13), one can manufacture the following annihilation and creation operators:

$$
\begin{aligned}
A_{f}(\vec{p}, \beta)= & \left(\frac{1}{2(2 \pi)^{D} f(\vec{p})}\right)^{1 / 2} \int_{x}\left\{f ( \vec { p } ) \left[e^{-i \vec{p} \cdot \vec{x}} \operatorname{ch}(g(\vec{p}, \beta))\left(\phi_{x}-\varphi_{x}\right)\right.\right. \\
& \left.\left.-e^{i \vec{p} \cdot \vec{x}} \operatorname{sh}(g(\vec{p}, \beta))\left(\tilde{\phi}_{x}-\varphi_{x}\right)\right]+i e^{-i \vec{p} \cdot \vec{x}} \operatorname{ch}(g(\vec{p}, \beta)) \Pi_{x}-i e^{i \vec{p} \cdot \vec{x}} \operatorname{sh}(g(\vec{p}, \beta)) \tilde{\Pi}_{x}\right\}
\end{aligned}
$$

and

$$
\begin{aligned}
A_{f}^{\dagger}(\vec{p}, \beta)= & \left(\frac{1}{2(2 \pi)^{D} f(\vec{p})}\right)^{1 / 2} \int_{x}\left\{f ( \vec { p } ) \left[e^{i \vec{p} \cdot \vec{x}} \operatorname{ch}(g(\vec{p}, \beta))\left(\phi_{x}-\varphi_{x}\right)\right.\right. \\
& \left.\left.-e^{-i \vec{p} \cdot \vec{x}} \operatorname{sh}(g(\vec{p}, \beta))\left(\tilde{\phi}_{x}-\varphi_{x}\right)\right]-i e^{i \vec{p} \cdot \vec{x}} \operatorname{ch}(g(\vec{p}, \beta)) \Pi_{x}+i e^{-i \vec{p} \cdot \vec{x}} \operatorname{sh}(g(\vec{p}, \beta)) \tilde{\Pi}_{x}\right\} .
\end{aligned}
$$

with the relations $\left[A_{f}(\vec{p}, \beta), A_{f}^{\dagger}\left(\vec{p}^{\prime}, \beta\right)\right]=\delta\left(\vec{p}^{\prime}-\vec{p}\right)$ and $A_{f}(\vec{p}, \beta) \mid \varphi, \beta>=0$. The thermo-particle creation and annihilation operators of the tilde system can be obtained from Eqs.(14) and (15) according to the rule of the tilde operation.

Letting the thermo-particle creation operator $A_{f}^{\dagger}(\vec{p}, \beta)$ act on the thermal vacuum Eq.(11) or (13), we can construct the one- and two-thermo-particle excited states ( at the frame of the mass center ) as

$$
\left|1>=A_{f}^{\dagger}(\vec{p}, \beta)\right| \varphi, \beta>
$$

and

$$
\left|2>=\int d^{D} \vec{p} \Sigma(\vec{p}) A_{f}^{\dagger}(\vec{p}, \beta) A_{f}^{\dagger}(-\vec{p}, \beta)\right| \varphi, \beta>,
$$

respectively, where $\Sigma(\vec{p})$ is the Fourier transform of the S-wave function of the two thermo-particles with $\Sigma(-\vec{p})=\Sigma(\vec{p})$. Of course, one can also manufacture the multi-thermo-particle states and the excited states of the thermal tilde system. Here, we discuss them no more. 
Next we are ready to calculate the FTGEP. From Ref. [8], the entropy operator is

$$
S=\int d p\left[A_{f}(\vec{p}) A_{f}^{\dagger}(\vec{p}) \ln \left(\operatorname{ch}^{2}(g(\vec{p}, \beta))\right)-A_{f}^{\dagger}(\vec{p}) A_{f}(\vec{p}) \ln \left(\operatorname{sh}^{2}(g(\vec{p}, \beta))\right)\right],
$$

and hence, the Helmholtz free energy operator reads

$$
F=\int_{x} \mathcal{N}_{Q}\left[\mathcal{H}_{x}\right]-\frac{1}{\beta} S
$$

(Actually $k_{B} S$ is the entropy). Using functional integration technique and the integral formula $\int_{0}^{\infty} \frac{2 a}{\sqrt{\pi}} e^{-a^{2} x^{2}} d x=1$, we obtain the average value

$$
<\varphi, \beta\left|\mathcal{N}_{Q}\left[V\left(\phi_{x}\right)\right]\right| \varphi, \beta>=\int_{-\infty}^{\infty} \frac{d \alpha}{2 \sqrt{\pi}} e^{-\frac{\alpha^{2}}{4}} V\left(\frac{\alpha}{2} \sqrt{J_{1}(g)-I_{1}\left(Q^{2}\right)}+\varphi_{x}\right)
$$

with the notation $J_{n}(g)=\int \frac{d p f(\vec{p}) \operatorname{ch}(2 g(\vec{p}, \beta))}{(2 \pi)^{D}(f(\vec{p}))^{2 n}}$. Thus making some functional integrations leads to the vacuum average value of the free energy

$$
\begin{aligned}
\mathcal{F}[\varphi, g, f ; \beta]= & <\varphi, \beta|F| \varphi, \beta> \\
= & \frac{1}{4} J_{0}(g)+\frac{1}{2}\left(\nabla \varphi_{x}\right)^{2}+\frac{1}{4} \int \frac{d^{D} \vec{p}}{(2 \pi)^{D}} \frac{p^{2}}{f(\vec{p})} \operatorname{ch}(2 g(\vec{p}, \beta))-\frac{1}{2} I_{0}\left(Q^{2}\right)+\frac{1}{4} Q^{2} I_{1}\left(Q^{2}\right) \\
& +\int_{-\infty}^{\infty} \frac{d \alpha}{2 \sqrt{\pi}} e^{-\frac{\alpha^{2}}{4}} V\left(\frac{\alpha}{2} \sqrt{J_{1}(g)-I_{1}\left(Q^{2}\right)}+\varphi_{x}\right) \\
& -\frac{1}{\beta} \int \frac{d p}{(2 \pi)^{D}}\left[\operatorname{ch}^{2}(g(\vec{p}, \beta)) \ln \left(\operatorname{ch}^{2}(g(\vec{p}, \beta))\right)-\operatorname{sh}^{2}(g(\vec{p}, \beta)) \ln \left(\operatorname{sh}^{2}(g(\vec{p}, \beta))\right)\right]
\end{aligned}
$$

which is a functional of the functions $\varphi_{x}, g, f$. When the field system Eq.(1) has a thermal equilibrium with the heat reservoir at $T \neq 0$, its free energy is absolutely minimized. So minimizing the free energy with respect to $f(\vec{p})$ ( the Fourier transform of function $f_{x y}$ ) and taking $\varphi_{x}=$ const. $=\varphi$, we obtain

$$
f(\vec{p})=f(p, \beta)=\sqrt{p^{2}+\mu^{2}(\varphi, \beta)},
$$

where

$$
\mu^{2}(\varphi, \beta)=\int_{-\infty}^{\infty} \frac{d \alpha}{2 \sqrt{\pi}} e^{-\frac{\alpha^{2}}{4}} V^{(2)}\left(\frac{\alpha}{2} \sqrt{J_{1}(g)-I_{1}\left(Q^{2}\right)}+\varphi\right)
$$

with $V^{(n)}(z)=\frac{d^{n} V(z)}{d z^{n}}=\int_{-\infty}^{\infty} \frac{d \Omega}{\sqrt{2 \pi}}(i \Omega)^{n} \tilde{V}(\Omega) e^{i \Omega z}$. Thus, we see that the variational procedure has enforced the function $f$ depend both upon $\varphi$ and upon $\beta$ through the function $g$.

Furthermore, regardless of the relation Eq.(22) and independent of the last variational procedure, one can minimize the free energy Eq.(21) with respect to $g(\vec{p}, \beta)$ and obtain

$$
g(\vec{p}, \beta)=g(p, \beta)=\ln \left(\frac{e^{\frac{1}{2} \beta f(p, \beta)}+1}{e^{\frac{1}{2} \beta f(p, \beta)}-1}\right) .
$$

( Here, in the minimized result, we have made $f(p, \beta)$ take the place of $f(\vec{p})$ ). Now the function $g$ is evidently related to $\beta$, owing to the variational procedure. This point is similar to Ref. [11, but different from Ref. [10], where the expression between $\theta$ and $\beta$ is artificially given ( $\theta$ there corresponds to $g$ here ). Substituting Eqs.(22) and (24) into Eq.(21), one has the FTGEP of Eq.(1)

$$
\begin{aligned}
\mathcal{V}_{T}(\mu, \beta, \varphi) \equiv & \mathcal{F}(\mu, \beta, \varphi)=\left.\mathcal{F}\left[\varphi=\text { const., } g_{x y}(\beta), f_{x y} ; \beta\right]\right|_{\mu^{2}(\varphi, \beta) \rightarrow \mu^{2}} \\
= & \frac{1}{2} J_{0}(g)-\frac{\mu^{2}}{4} J_{1}(g)-\frac{1}{2} I_{0}\left(Q^{2}\right)+\frac{1}{4} Q^{2} I_{1}\left(Q^{2}\right) \\
& +\int_{-\infty}^{\infty} \frac{d \alpha}{2 \sqrt{\pi}} e^{-\frac{\alpha^{2}}{4}} V\left(\frac{\alpha}{2} \sqrt{J_{1}(g)-I_{1}\left(Q^{2}\right)}+\varphi\right) \\
& -\frac{1}{\beta} \int \frac{d p}{(2 \pi)^{D}}\left[\operatorname{ch}^{2}(g(\vec{p}, \beta)) \ln \left(\operatorname{ch}^{2}(g(\vec{p}, \beta))\right)-\operatorname{sh}^{2}(g(\vec{p}, \beta)) \ln \left(\operatorname{sh}^{2}(g(\vec{p}, \beta))\right)\right] .
\end{aligned}
$$


In this equation, " $\rightarrow$ " means that $\mu$ takes the place of $\mu(\varphi, \beta)$. Because of the nature of the minimizing procedure, $\mu$ should be chosen from the non-zero root of Eq.(23) and two end points of the range $0<\mu<\infty$ so that $\mathcal{V}_{T}[\mu, \beta, \varphi]$ is an absolute minimum. Besides, sometimes the non-zero solution of Eq.(23) is multi-valued, and so in that case, the suitable root should be decided according to the stability condition

$$
\begin{aligned}
& \frac{\partial^{2} \mathcal{F}[\varphi, g, f ; \beta]}{\left(\partial \mu^{2}\right)^{2}} \\
& =\frac{1}{8} J_{2}(g)\left[1+\frac{1}{8} J_{2}(g) \int_{-\infty}^{\infty} \frac{d \alpha}{2 \sqrt{\pi}} e^{-\frac{\alpha^{2}}{4}} V^{(4)}\left(\frac{\alpha}{2} \sqrt{J_{1}(g)-I_{1}\left(Q^{2}\right)}+\varphi\right)\right]>0 .
\end{aligned}
$$

For terms comprising $J_{n}(g)$ 's and $I_{n}\left(Q^{2}\right)$ 's in Eqs.(23), (25) and (26), we can write them as

$$
\begin{aligned}
& \frac{1}{2} J_{0}(g)-\frac{1}{4} \mu^{2} J_{1}(g)-\frac{1}{2} I_{0}\left(Q^{2}\right)+\frac{1}{4} Q^{2} I_{1}\left(Q^{2}\right) \\
&= \frac{1}{2}\left[I_{0}\left(\mu^{2}\right)-I_{0}\left(Q^{2}\right)\right]-\frac{1}{4} \mu^{2} I_{1}\left(\mu^{2}\right)+\frac{1}{4} Q^{2} I_{1}\left(Q^{2}\right)+C_{0}-\frac{\mu^{2}}{2} C_{2}, \\
& J_{1}(g)-I_{1}\left(Q^{2}\right)=I_{1}\left(\mu^{2}\right)-I_{1}\left(Q^{2}\right)+2 C_{1},
\end{aligned}
$$

and

$$
\begin{aligned}
& \frac{1}{\beta} \int \frac{d p}{(2 \pi)^{D}}\left[\operatorname{ch}^{2}(g(p, \beta)) \ln \left(\operatorname{ch}^{2}(g(p, \beta))\right)-\operatorname{sh}^{2}(g(p, \beta)) \ln \left(\operatorname{sh}^{2}(g(p, \beta))\right)\right] \\
& =C_{0}-\frac{1}{\beta} \int \frac{d^{D} \vec{p}}{(2 \pi)^{D}} \ln \left(1-e^{-\beta f(p, \beta)}\right),
\end{aligned}
$$

where $C_{n} \equiv \int \frac{d^{D} \vec{p}}{(2 \pi)^{D}} \frac{f^{1-n}(p, \beta)}{e^{\beta f(p, \beta)}-1}$. Obviously, it is perhaps hard for $C_{0}, C_{1}$ and $C_{2}$ to have simple analytic expressions, but they are all finite. Moreover, for the case of $D<3$, although $I_{n}\left(\mu^{2}(\beta, \varphi)\right)$ 's and $I_{n}\left(Q^{2}\right)$ 's are divergent, the divergences can all cancelled each other in the above three expressions. So when $D<3$, Eqs.(23),(25) and (26) have not any divergence, and accordingly no further renormalization procedures are needed. This indicates that the finite temperature field theory with Eq.(1) is renormalizable for the case of $D<3$, at least within the framework of the FTGWFA.

Now we are in a position for calculating one- and two-thermo-particle energies. For thermo-particle excited states, thermofield dynamics uses the following Hamiltonian $\hat{\mathrm{H}}$ of the combined system of the $T=0$ field system and its tilde partner

$$
\mathcal{N}_{Q}[\hat{H}] \equiv \int_{x}\left\{\mathcal{N}_{Q}\left[\mathcal{H}_{x}\right]-\mathcal{N}_{Q}\left[\tilde{\mathcal{H}}_{x}\right]\right\}
$$

and each thermo-particle excited state is the eigenstate of $\mathcal{N}_{Q}[\hat{H}]$. Similar to the calculation of the FTGEP, using functional integration technique, one can obtain the one-thermo-particle energy

$$
m_{1}(\varphi, \beta)=\frac{<1\left|\mathcal{N}_{Q}[\hat{H}]\right| 1>}{<1 \mid 1>}=\sqrt{p^{2}+\mu^{2}(\varphi, \beta)}
$$

and the two-thermo-particle energy

$$
\begin{aligned}
m_{2} & \equiv \frac{<2\left|\mathcal{N}_{Q}[\hat{H}]\right| 2>}{<2 \mid 2>} \\
& =\frac{2 \int d^{D} \vec{p} \Sigma^{2}(p, \beta) f(p, \beta)+\frac{\nu^{(4)}(\varphi, \beta)}{8(2 \pi)^{D}} \int \frac{d^{D} \vec{p} \Sigma(p, \beta)}{f(p, \beta)} \int \frac{d^{D} \vec{p} \Sigma(p, \beta) c h(2 g(p, \beta))}{f(p, \beta)}}{\int d^{D} \vec{p}[\Sigma(p, \beta)]^{2}},
\end{aligned}
$$

where

$$
\nu^{(n)}(z, \beta)=\int_{-\infty}^{\infty} \frac{d \alpha}{2 \sqrt{\pi}} e^{-\frac{\alpha^{2}}{4}} V^{(n)}\left(\frac{\alpha}{2} \sqrt{J_{1}(g)-I_{1}\left(Q^{2}\right)}+z\right)
$$


Minimizing $m_{2}$ with respect to $\Sigma(p, \beta)$ leads to a second kind of Fredholm integral equation about $\Sigma(p, \beta)$ [15], and solving it, we have

$$
\Sigma(p, \beta)=\frac{C}{f(p, \beta)\left(2 f(p, \beta)-m_{2}\right)}\left[\operatorname{ch}(2 g(p, \beta))+\frac{\int \frac{d p c h^{2}(2 g(p, \beta))}{f^{2}(p, \beta)\left(2 f(p, \beta)-m_{2}\right)}}{1+\frac{\nu^{4}(\varphi, \beta)}{16(2 \pi)^{D}} \int \frac{d p c h(2 g(p, \beta))}{f^{2}(p, \beta)\left(2 f(p, \beta)-m_{2}\right)}}\right]
$$

with $C$ some normalization constant. Substituting the last expression into Eq.(29) and noting the normalization of $\mathrm{C}$, one can compute the energy $m_{2}$. From Eq. $(28)$, one can see that $\mu(\varphi, \beta)$ is just the mass of one thermal particle. Thus, Eq.(23) can be used for calculating the mass of a single thermo-particle. Moreover, the two terms in Eq.(29) can be interpreted as the kinetic energy of the two constituent thermal particles and their interacting energy, respectively. One can perhaps dicuss thermo-particle bound states and scattering states with the help of Eq.(29) [3] (1993).

In this letter, we have developed the GWFA of thermofield dynamics in $(D+1)$ dimensions, and calculated the FTGEP, one- and two-thermo-particle-state energies of the system Eq.(1). Equations (25),(28) and (29) with some relevant relations are convenient for an investigation of any concrete model involved into the class of Eq.(1) (including some bosonized models in condensed matter physics [16] ). When Eq.(25) is applied to $\lambda \phi^{4}$ and $\phi^{6}$ models, one can obtain the same, corresponding results as in Ref. [7] (Roditi, Okopinska, Hajj and Cea ). Again, contrasting the results in Ref. [17] with ones here, and noting the above-mentioned analysis about $J_{n}(g)$ 's and $I_{n}\left(Q^{2}\right)$ 's, one can have the replacement rule between the FTGEP and GEP which indicated in Ref. [7] $\left(J_{n}(g) \rightarrow I_{n}\left(\mu^{2}(\varphi)\right)\right)$. Still, the $T=0$ limit of each of Eqs.(25), (28) and (29) is identical with that in Ref. [17]. Moreover, employing Eqs.(5), (6), (9) and (10), one can find that the exponential factor in Eq.(11) is just field-operator form of a thermal Bogoliubov transformation, and therefore conclude that the TDGWFA is equivalent to the TDBTT. In fact, when $I_{n}\left(Q^{2}\right)^{\prime} s$ are deleted, Eq.(25) can give the non-renormalized equation Eq.(33) of Ref. [11]. Besides, if a non-uniform background field is considered, the term $\frac{1}{2}\left(\nabla \varphi_{x}\right)^{2}$ will be added to Eq.(25) with $\varphi_{x}$ replacing $\varphi$, and then one can have the following equation by $\frac{\partial\left(\mathcal{V}_{T}\left[\mu, \beta, \varphi_{x}\right]\right)}{\partial \varphi_{x}}=0$

$$
\nabla^{2} \varphi_{x}-\int_{-\infty}^{\infty} \frac{d \alpha}{2 \sqrt{\pi}} e^{-\frac{\alpha^{2}}{4}} V^{(1)}\left(\frac{\alpha}{2} \sqrt{J_{1}(g)-I_{1}\left(Q^{2}\right)}+\varphi_{x}\right)=0
$$

the $T=0$ limit of which is identical with that of Ref. [17]. This equation can be used for considering quantum thermal solitons. Basing on thermal soliton solution, Eqs.(28) and (29) can perhaps give excited-state results of thermo-solitons. Of course, when the non-uniform background field is considered, the discussion about the difference between the BTT and GWFA in Ref. [17] is valid at the finite temperature case. Finally, the GWFA of thermofield dynamics can be generalized to the time-dependent case so as to investigate dynamics of statistical mechanical systems.

\section{ACKNOWLEDGMENTS}

This work was finished at the Abdus Salam International Centre for Theoretical Physics (ICTP), and the author would like to thank the Abdus Salam ICTP for hospitality. This project was supported by the President Foundation of Shanghai Jiao Tong University.

[1] Schiff L I 1963 Phys. Rev. 130458 ; Cornwall J M, Jackiw R and Tomboulis E 1974 Phys. Rev. D 102428 ; Barnes T and Ghandour G I 1980 ibid. 22924.

[2] Bardeen W A, Moshe A and Bander M 1994 Phys. Rev. Lett. 521188 ; Darewych J W, Horbatsch M and Koniuk R 1985 ibid. 542188 ; Polley L and Pottinger D E L ed. 1987 Proceedings of the international workshop in Variational calculations in Quantum field theory, Wangerooge, West Germany, 1-4 September,1987, (Published by World Scientific, Singapore); Jackiw R 1989 Field Theoretic Results in the Schrödinger Representation (Troisieme Cycle De La Physigue, En Suisse Romande); Ni G J and Chen S Q 1995 Levison Theorem, Anomaly and the Phase Transition of Vacuum, Shanghai Scientific and Technical Publishers, Chapter 7 (in Chinese).

[3] Lu W F, Xu B W and Zhang Y M 1993 Phys. Lett. B 309109 ; Lu W F, Ni G J and Wang Zh G 1998 J. Phys. G 24673 and references therein.

[4] Stevenson P M 1984 Phys. Rev. D 301712 ; 1985 ibid. 321389. 
[5] Cea P and Tedesco L 1997 Phys. Rev. D 554967 ; Kümmel H G Jan. 25th 1998 Preprint, Post-Gaussian Approximation to the Vacuum Sector of the $(1+1)$ Dimensional Sine-Gordon Model; Lee G H, Lee T H and Yee J H 1998 E-print hep-th/9804094.

[6] Alexander F J and Eyink G L 1997 Phys. Rev. Lett. 78 1; Gogolin A O 1993 ibid. 712995 ; Dean D S and Lancaster D 1996 ibid. 773037 .

[7] Roditi I 1986 Phys. Lett. B 169264 ; Okopinska A 1987 Phys. Rev. D 362415 ; Hajj G A and Stevenson P M 1988 ibid. 37413 ; Lou S Y and Ni G J 1991 Science in China A 3468 ; Cea P and Tedesco L 1997 J. Phys. G 23135.

[8] Takashi Y and Umezawa H 1975 Collective Phenomena 255 ; Umezawa H, Matsumoto H and Tachiki M 1982 Thermo Field Dynamics and Condensed states (North-Holland, Amsterdam).

[9] Das A 1997 Finite Temperature Field Theory (World Scientific, Singapore).

[10] Roditi I 1986 Phys. Lett. B 17785 .

[11] Mishra A and Mishra H 1997 J. Phys. G 23143.

[12] Boccara N 1990 Functional Analysis - An Introduction for Physicists (Academic Press INC.).

[13] Coleman S 1975 Phys. Rev. D 112088 ; Chang S J 1976 Phys. Rev. D 132778.

[14] Bogoliubov N N and Shirkov D V 1983 Quantum Fields, trans. by Portecorvo, Appendix IV (the Benjamin/cummings Publishing Company, Inc.); Kirzhnits D A 1967 Field Theoretical Methods in Many-body Systems, Appendix B (Pergamon Press).

[15] Tricomi F G 1975 Integral Equations, (Interscience Publishers, Inc., New York) .

[16] Fradkin E 1991 Field Theories of Condensed Matter System, Addison-Wesley Publishing Company .

[17] Lu W F, Chen S Q and Ni G J 1995 J. Phys. A 287233. 